\title{
Keep Calm and Cuddle on: Social Touch as a Stress Buffer
}

\author{
India Morrison ${ }^{1}$
}

Received: 5 February 2016 / Revised: 2 August 2016 / Accepted: 3 August 2016 /

Published online: 29 August 2016

C The Author(s) 2016. This article is published with open access at Springerlink.com

\begin{abstract}
Recent evidence from neurophysiology and human functional neuroimaging has given rise to the hypothesis that social, affective touch belongs to a distinct category of tactile experience. Such hedonic and rewarding touch is proposed to operate mainly in the domain of social interactions and relationships. Social touch may play a functional role in the physiological regulation of the body's responses to acute stressors and other short-term challenges. In this perspective, touch can "buffer" disadvantageous physiological effects of potentially inefficient or maladaptive responses. This review outlines the evidence for such a role, as well as the neural pathways that may support it. Direct evidence for touch as a physiological regulator is strongest for systems that underlie the maintenance of physical proximity to conspecifics in a variety of circumstances. For example, mammalian social physical contact involves social thermoregulatory processes like huddling and snuggling, which also rely on tactile thermosensory and somatosensory pathways. There is also good evidence that touch systems contribute to preventing social separation and facilitating the re-instatement of contact following social separation. Finally, prosocial touch, such as allogrooming and consolation, may utilize some of the same neural pathways as other, non-social means of stress regulation. Social touch may thus serve as part of a system for regulation of responses to acute stressors, "extended" to include the physiological effects of social interactions.
\end{abstract}

Keywords Affective touch $\cdot$ Stress buffer $\cdot$ Neuropeptides $\cdot$ Grooming $\cdot$ Social thermoregulation

\section{Social touch and regulation of physiological responses}

Touch often acts as a pivot-point for emotion in social interactions. Recently, neuroscientific research has begun to focus more intensively on this social and affective dimension

India Morrison

india.morrison@liu.se

1 Center for Social and Affective Neuroscience (CSAN), Department of Clinical and Experimental Medicine, University of Linköping, Psychiatry Building, entrance 27, floor 9, Linköping, Sweden 
of touch. Converging evidence suggests that affective touch is a distinct domain, distinguishable on the behavioral and neural levels from so-called "discriminative" aspects of touch (Morrison et al. 2010, McGlone et al. 2014, Morrison 2016a)-such as rapid stimulus localization and fine-grained tactile perception. However, the task remains to characterize what this distinction implies from a functional perspective. Does affective touch have a unique, specific functional role? To explore this question, this review goes beyond a focus on the sensory encoding of touch, to examine the neural mechanisms by which affective, social touch might influence how the body reacts and adjusts to events in the world, especially those involving short-term stresses and bodily challenges.

There are many domains of bodily challenge and stress, but this review outlines evidence concerning three broad ways in which touch is likely to be relevant in social interactions: 1) social thermoregulation; 2) the regulation of physical proximity to others; and 3) prosocial touch (eg allogrooming and consolation behavior). In one respect or another, each of these domains involves the organism changing or maintaining physical proximity in social interactions and relationships, particularly those in which close proximity has general benefits for safety (whether from an immediate or an evolutionary perspective). On the level of physiology and neuroanatomy, each of these domains also involves regulation of responses to external events, usually via brain pathways. The ultimate role of this neural regulation is to keep a stable balance among the body's multiple interacting physiological systems during the usual fluctuations of living. Here, stability is critical from the standpoint of efficient energy expenditure, and central to the idea of "allostasis" (Sterling 1988; McEwen 1998; Schulkin 2011). Allostatic regulation aims to keep the body's systems within a range in which energy is neither wasted nor overconserved in its overall responses to changing conditions.

This review also identifies potential key aspects of the underlying neurocircuitry that might allow affective touch to affect responses to stress and other challenges to the body. Structures in the brain and brainstem provide interfaces between incoming sensory information and integrated higher-level information such as emotion, memory, and prospection. Because these circuits encompass crucial brain regions with connections to and from brainstem autonomic control centers, they provide candidate neurocircuitry for affective touch to influence regulation of physiological responses to the "slings and arrows" of everyday life.

\section{Stress Responses and "Stress Buffering"}

If social touch influences how the body reacts to challenges, how might this be mediated? In a very general sense, the physiological relationship between bodily challenges and bodily reactions can be viewed in terms of "stressors" and "stress responses." Social touch may tap into the complex dance between the bodily systems that defend the organism against external challenges or stressors - stress responses and those that calibrate the stress responses to avoid spending too much or too little energy. Such calibration mechanisms are referred to here as "stress buffers." This section sketches a conceptual overview of stress responses and stress buffering in order to further explore the possibility that affective, social touch plays a stress buffering role.

The term "stress responses" usually refers to activation of specific systems in the body that allow prompt, sometimes life-preserving, reactions to events. These systems 
invigorate responses to short-term challenges by making energy quickly available to the body's cells, as well as facilitating behavioral and bodily adjustments to such challenges. For example, neurochemical cascades in the hypothalamic-pituitary-adrenal (HPA) axis help to mobilize glucose in muscle and organ cells to produce energy (McEwen 1998). The sympathetic-adrenal-medullary (SAM) axis can similarly jumpstart cardiac and respiratory responses in order to facilitate fast oxygen delivery to muscles and other cells (McEwen 1998; Gunnar and Hostinar 2015). In these stressresponse systems, the body's efficient use of its energy economy and the effective, adaptive modulation of behavior are key.

Under typical circumstances, such stress responses are adaptive. But they can also have a dangerous flip side (Valentino and Van Bockstaele 2015). For example, some HPA and SAM stress responses occur at the expense of important immune and inflammatory processes. In the longer term, chronic activation can impair growth and healing, flatten stress responses, and even shrink hippocampal neurons involved in learning (Miller et al. 1994, Sapolsky 1994; Herman and Cullinan 1997; Sapolsky 2005). Further, because the body is a complex mesh of dynamically interacting systems, blunted or dysfunctional responses can also create asymmetric pressures on other systems, which often heighten their own activity in response to decreased or badlycoordinated stress reactions elsewhere in the body (McEwen 2004). Stress responses are best suited to meet acute, one-off challenges, for example when one is facing aggression, braking to avoid a car accident, or running from a bear. In such emergencies stress responses are cost-effective, even considering the short-term negative impact on the body. Stress responses are therefore not a bad thing in themselves, but under some circumstances, the efficiency of these systems - in terms of energy expenditure, efficacy, and flexibility of response - can be compromised (eg, Schulkin 2011).

The timing of stress responses is also vital. The brain's networks integrate information from multiple sources, domains, and levels, and exert descending control over peripheral regulatory systems. The scope for the body's peripheral systems to anticipate and predict challenges before they occur is limited, compared to that of the brain. Through a wider range of learning and predictive abilities, the brain can engage stress responses in anticipation of a stressor. That is, they can be proactive as well as reactive (Herman et al. 2003; IJzerman et al. 2015). It may indeed be the case that the anticipatory or proactive category of stress responses carries especial risk of maladaptation under conditions of chronic stress (Herman et al. 2003). Proactive stress responses can also involve anxiety, in which activation of stress responses extend beyond the immediate context. Anxiety-related HPA and SAM activation is associated with an increased risk of cardiac disease (Ouakinin 2016).

Systems such as the HPA and SAM axes operate most efficiently within a certain "zone", and less efficiently outside this zone. (This general idea has been discussed elsewhere, eg McEwen 1998). This is likely to be true of most systems which keep the body functioning under changing circumstances. For example, thermoregulatory systems (discussed in the next section) protect the body's tissues from straying outside a specific temperature range and getting too hot or too cold. The general energyefficiency-centered range in allostatic regulatory systems will be referred here to as an "Efficiency Zone" (EZ; see Fig. 1). The term "Efficiency Zone" is coined here as a general label for processes and effects that have been noted and discussed in the literature, for example in Sterling (1988). 
Physiological responses to stress and challenge are expected to be within an EZ under these conditions:

1) responses are easily modulated and adaptive, for example cortisol release showing a sharp, well-timed response.

2) responses return to baseline after the stressful event, without becoming blunted or attenuated.

3) responses are acute and infrequent.

4) responses are well-tuned during a developmental sensitive period (eg, in infancy or early childhood, see Hostinar et al. 2014).

So when stress responses fall outside the extremes of an EZ, the costs of activation (or lack thereof) can begin to outweigh the benefits. For example, frequent dilation of blood vessels in a SAM-mediated response to stress, or their frequent constriction in chronic cold exposure (Castellani and Young 2016), can result in reduction in vessel elasticity and thus reactivity. Outside the EZ, then, risks from within can begin to overshadow risks from without.

However, in maintaining allostatic balance, the body may enlist "buffers" that anticipate and attenuate responses that fall outside the EZ. Overall, the stress buffer idea can be operationalized as responses that offset or mitigate the negative effects of responses outside the EZ, or those that provide some check against the loss of efficiency. Some of these buffering mechanisms may have a genetic basis, as suggested by evidence that certain gene variants can confer a degree of protection, or "resilience," to stress ( $\mathrm{eg}$, Osorio et al. 2016). Some may have developed via natural selection in evolutionary history, such as the comparatively phylogenetically recent ability of the parasympathetic vagal nerves to mitigate sympathetic arousal (Porges 2007). Finally, some buffers may arise from more plastic mechanisms based on learning, experience, and early development. This can involve, for example, developmental time windows (Hostinar et al. 2014), changes in gene transcription and expression (Champagne et al. 2008, Burbridge et al. 2016), and even some forms of gene competition within an individual genome

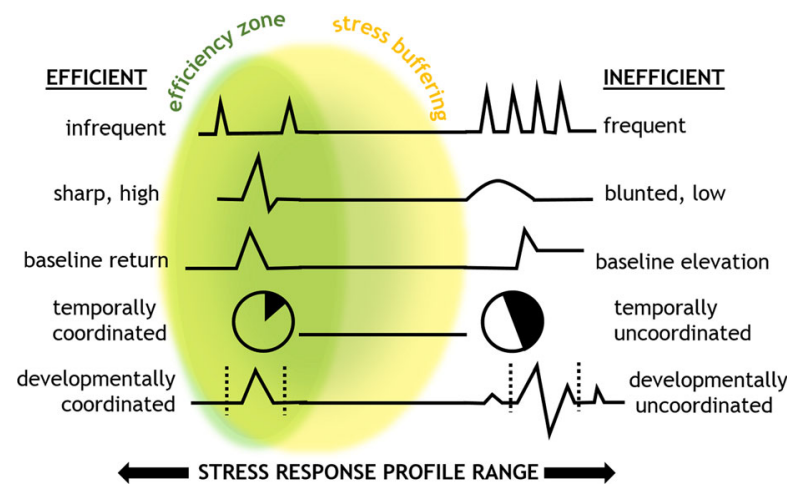

Fig. 1 Schematic illustration of physiological stress responses along an axis of efficiency. Different forms of response on the left extreme are in the efficiency zone (EZ; green), providing flexible, adaptive, and energyefficient responses to stress challenges. The right extreme represents dysfunctional responses, which carry the danger of maladaptivity and the potential of deleterious consequences. Stress buffering mechanisms are likely to have most relevance at the right edge of the EZ (yellow). A stress buffering mechanism mitigates or prevents energy efficiency losses among regulatory systems, while also allowing adequate responses to external challenges 
(Champagne et al. 2009). This broad scope for buffering mechanisms gives rise to a tentative generalized definition: a stress buffer is any mechanism or process that mitigates, attenuates, offsets, or prevents energy efficiency losses among regulatory systems, while remaining adequately responsive to external challenges.

If social touch indeed plays such a stress-buffering role, this may involve an interplay between conserved mechanisms of physiological regulation, and those more plastic pathways that change during the course of an individual life, providing flexibility of response and sensitivity to relevant short-term events. It is therefore reasonable to predict that social touch could modulate neural systems which protect bodily safety via regulation of physiological responses, such as the comparatively well-studied systems of cortisol release and regulation. Any such buffering role for affective, social touch might apply to anything between acute, reactive responses within the EZ (eg, a bad day at the office), or straying outside the EZ (eg, chronic anxiety). In a sense, then, our stress-regulatory systems could even extend outside the body envelope to include other people. But what is the current evidence that it does so?

\section{Keeping Warm: Social Thermoregulation and Social Touch}

Cell membranes, enzymes, and other important ingredients of our body tissues have an exquisitely narrow temperature range in which they can survive and operate. Mammals can endogenously regulate body temperature through endothermogenic processes, in which the body produces its own heat by shivering or metabolizing fat. A common thermoregulation strategy shared by mammals and other taxa is to move around and change behavior to exploit heat sources or to cool off. Part of this behavioral thermoregulation strategy involves exploiting local heat sources, thereby cutting the metabolic costs of endothermoregulation. Mammals - especially social mammals - take advantage of the fact that conspecifics can double as cheap space heaters, providing decisive gains in the efficient regulation of energetic resources. In most altricial mammals, social thermoregulation has its greatest impact during the period immediately following birth.

Huddling in infant mammals is a major type of social thermoregulation that allows reduction of heat loss while lowering energetic metabolic costs of endothermoregulation (Gilbert et al. 2007, Morrison et al. 2008, Nakamura and Morrison 2008a, b; Gilbert et al. 2010). Huddling in newborn porcupines (Hystrix africaeaustralis), for example, lowers the critical temperature at which they can effectively thermoregulate by endogenous means (Haim et al. 1992). Importantly, these decreases enable gains in energy allocation for other important processes, such as growth, healing, and cell repair. In many mammal species that give birth to multiple offspring at the same time, such as rat litters, the offspring huddle in a pile. In nonhuddling mammals such as primate parent-offspring dyads, the equivalent may be snuggling and cuddling, in which body surface contact is increased alongside changes in movement and pressure.

Yet huddling is by no means a static, passive affair. Rather, it involves active behavior to maximize its effects, which results in continual movement patterns as individuals nuzzle their way to the center, displacing and being displaced by littermates (Blumberg et al. 1992, Gilbert et al. 2007, Gilbert et al. 2012). In rats, individual pups dynamically 
adjust their thermal gains and losses by changing the amount of surface area exposed to the outer, colder perimeter of the huddle - outer pups tunnel inwards to minimize exposure and increase warmth, while the pups on the inside either get displaced or move outwards (Alberts 2007). The huddle is thus a group-level phenomenon, in which the dynamics are most parsimoniously accounted for by considering the aggregate surface area of the huddle itself, rather than that of any given individual pup. Another important thermodynamic influence on the huddle is the mother. As Alberts (2007, page 25) vividly describes: "For the pups, the dam is a mobile feast of experiences, for the dam's body approaches and settles upon them, often encircling the entire litter, and it remains in place while they root, squirm, shift, probe, and push. It undulates with each breath. Throughout such contact, conductive heat exchange is maintained."

Crucially for the matter of social touch, this squirming, shifting, probing, and pushing also generates predictable, stable signatures of somatosensory stimulation. This means that thermoregulatory behavior and somatosensory stimulation are linked, both in temporal and contextual terms. This linkage raises the possibility that the underlying mechanisms of thermoregulation and somatosensation may have themselves become linked in the mammalian phylogenetic past. That is, pathways involved in social thermoregulation may have evolved to use conspecific touch patterns as shorthand for "warm and close." This possibility also carries an important implication that such temporal and contextual shorthand may manifest in regulatory neural shortcuts in which "warm and close" states can be instigated by social touch alone, rather than requiring a cycle of behaviorally-mediated physical warmth restoration following actual temperature decreases.

\section{Afferent Nerve Fibers: Temperature and Touch Sensitivity}

How might the link between social temperature and social touch be implemented neurally? On the level of peripheral nerve signaling, the common denominator between thermosensation and somatosensation is a class of functionally specialized nerve fibers in the skin called cutaneous $\mathrm{C}$ afferents. Some $\mathrm{C}$ afferent subtypes are cold-sensitive and signal decreases in skin temperature. This signaling reaches brainstem regulatory centers that can ultimately result in shivering or non-shivering thermogenesis. For example, in newborn mammals, non-shivering thermogenesis in response to cold can instigate the burning of energy-rich brown fat (brown adipose tissue or BAT; Ryu et al. 2015). Though the physiology is less well understood (but see Farrell and Alberts 2007), signals from cold-sensitive $C$ afferents to the brain may also result in changes in behavior to seek external heat sources, perhaps including the wriggling and moving involved in huddling.

Crucially, this wiggling and moving, involving gentle skin indentation and dynamic stimulation moving over skin, would be adequate to activate a further subtype of $\mathrm{C}$ afferent. The "tactile C" (or CT) afferent is predominantly mechanosensitive, responding optimally to gentle, moving touch at around $32{ }^{\circ} \mathrm{C}$ (Vallbo et al. 1999, Wessberg et al. 2003, Loken et al. 2009, Ackerley et al. 2014). In humans, CT afferents' preferred stimulus profile corresponds well to a gentle caress at skin temperature. Human CT afferents also display a "tuning curve" for caressing speeds that people find most pleasant (Loken et al. 2009, Morrison et al. 2011, Ackerley et al. 2014). The 
"social touch hypothesis" (Morrison et al. 2010, Olausson et al. 2010) postulates that CT afferents respond to the range of tactile stimulus features that is most likely to occur during close social interactions, and has an impact on behavior. Computationally, CTs may even act as a "middle pass" information filter (Morrison 2016b), selectively conveying to the brain information about those stimuli that are most likely to be socially or affectively relevant, on the basis of their dynamic and thermal properties. Importantly, the neural pathway that all $\mathrm{C}$ afferents follow to the brain has been classed as an "afferent limb" of the sympathetic nervous system (Craig 2003). This indirect evidence implies a relationship with the regulation of bodily states, for example those which influence heartbeat, breathing, and muscle readiness (Craig and Zhang 2006; Seth and Critchley 2013). In this perspective, some $\mathrm{C}$ afferent types may be wired to affect bodily regulation for touch, just as other subtypes of $\mathrm{C}$ afferents affect bodily regulation in response visceral signals from $\mathrm{C}$ afferents in the gut, or pain from nociceptive $\mathrm{C}$ afferents throughout the body (see Björnsdotter et al. 2010).

Because $\mathrm{C}$ thermoregulatory and mechanosensitive $\mathrm{C}$ afferents are functionally, anatomically, and phylogenetically close, they may interact with similar and even overlapping brain areas. This implies that somatosensory input from CT mechanoafferents has the potential of influencing classically thermoregulatory output via these shared or overlapping brain relays, which use sensory information to shape responses to changes in the environment. If this overlap includes circuitry that mediates thermally-driven contact-seeking behavior, it suggests that touch could be "plugged in" to the machinery of social thermoregulation discussed in the previous section. And at least in principle, that opens up an opportunity for the concomitant C-mediated somatosensory signaling during snuggling to become incorporated in the circuitry that influences thermally-driven contact-seeking behavior. Somatosensory stimulation optimal for activating CT nerves might even be able to indicate the likelihood of proximity to a conspecific, and help to drive behavior that proactively maintains that proximity during huddling or snuggling - even in the absence of more extreme, thermal changes needed to trigger reactive thermoregulatory behavior via $\mathrm{C}$ thermoreceptors. So because wiggling, moving conspecifics are also heat sources, somatosensation can not only provide a "most likely nice and warm" signal, but also a "most likely close to mom and littermates" signal, free with the package.

In principle, mechanosensitive $\mathrm{CT}$ afferents could have branched off in evolution from broadly thermoreceptive $\mathrm{C}$ afferents, differing in selectivity while remaining quite related in terms of functional neuroanatomy. Though this proposal is entirely speculative, such a tuning shift is plausible because cutaneous sensory $\mathrm{C}$ afferents in rodents have been observed to adjust the molecular receptor profiles of the neuron according to species-level environmental pressures (Gracheva and Bagriantsev 2015). Gene regulation mechanisms determine specific response profiles of $\mathrm{C}$ afferent nerve subtypes by differential expression of receptor channels (Ma 2010; Lou et al. 2013) both in development and phylogeny. So the selectivity of sensory neurons can be modified by turning "on" or "off" the expression of certain cell receptor channels (Ma 2010; Prescott et al. 2014) - in this case, perhaps shifting a subpopulation of $\mathrm{C}$ afferent nerves from being broadly thermoreceptive to broadly mechanoreceptive.

In summary, thermo- and somato-sensation share a common feature in a context of huddling, snuggling, or cuddling. Each essentially indexes an unexposed, secure environment, not too far away from warm, protective conspecifics. With this neural 
system as an evolutionary platform, it becomes possible to behaviorally regulate proximity to non-related conspecifics too, as well as preserving the underlying neurocircuitry from infancy to adulthood (enabling cuddling with a mate, for example). These processes can then ultimately serve to signal benefits of social proximity beyond the thermoregulatory domain, such as reduced risk for predation exposure. Social touch would thus become an energy-efficient way to sound the "all clear" to the central nervous system with respect to metabolically expensive stress responses and threat vigilance (see also Porges 2007). Conversely, it suggests a plausible neural means by which stress or anxiety could trigger a motivation to seek proximity in order to dampen HPA or SAM arousal, just as being cold can drive behavior to huddle close to others in order to restore warmth.

\section{Better together: $\mu$-Opioids and Oxytocin}

One of the primary protective benefits of sticking together with conspecifics is that threats from predation, cold, and hunger become less likely to affect an individual in a group, making the regulation of proximity to others a vital force in social living. Involuntary separation or isolation frequently triggers alarm and distress (Panksepp 1998), even in nonmammalian species. For example, in zebrafish (Danio rerio), stimulation of an isolated fish's lateral line tactile sensors, mimicking currents created by the school, reduces fear behavior and the emission of a stress-related signaling hormone (Schirmer et al. 2013). Proximity regulation both across and within taxa operates through multiple possible channels, at different levels, and for different reasons.

On a general level, proximity regulation can be thought about in terms of rough analogies with office supplies. Some forms are like rubber bands, yanking conspecifics back together if they become separated. Other forms act more like glue, keeping conspecifics stuck together to maintain a status quo of close proximity. There has been growing evidence over the past few decades that involuntary separation increases the motivation for seeking proximity, providing a "rubber-band-like" dynamic for restoring contact. Similarly, some more "gluelike" mechanisms of bonding may operate by reducing the motivation for voluntary straying, perhaps reinforcing the energetic and subjective rewards of remaining within the EZ. By extension, being away from others and outside the EZ can increase the motivation for social touch; and social touch can also make it more attractive to stay close in the first place.

Infant mammals become distressed when separated from their parents or close caregivers, and their distress behaviors (such as vocalizing) are reduced upon reunion (Panksepp et al. 1978b, Hofer et al. 1993, Panksepp et al. 1997). An influential model of the mechanism by which this occurs places an important neural receptor type, the $\mu$ opioid receptor (MOR), at center stage. Panksepp et al. (1978a, b)"Brain Opioid Theory of Social Attachment' (BOTSA) postulated that social, affiliative behaviors are steered by the same basic systems that allow the perception and alleviation of pain (Panksepp et al. 1978a). In this model, social separation involves a pain-like neurochemistry, which can be blocked when neurohormones called beta-endorphins bind to MOR receptors in the brain during social contact. Multiple lines of evidence suggest that this may alleviate the aversive subjective effects of isolation (Panksepp et al. 1997, Nelson and Panksepp 1998). 
In a sense, then, social isolation in the BOTSA model is akin to opioid withdrawal. Distress and contact-seeking motivation increase during separation, whereas reunion brings a surge of endorphins to occupy brain MOR receptors. This system may have originated as a "reactive" mechanism to change behavior under conditions of accidental, involuntary separation. Infant macaque monkeys (Macaca mulatta) that carry a slight difference in a gene (OPRM1), which increases the MOR receptor's binding affinity to endogenous opioids, are more likely to stick close to their mothers when very young, and more likely to engage in affiliative behaviors with their mothers after separations when they are older (Barr et al. 2008). Similar findings have been seen in free-ranging macaques (Higham et al. 2011) and adult humans (Troisi et al. 2011). Importantly, however, the feelings and behaviors dependent on MORs in a social context probably depend heavily on the state and even the species of the organism (Loseth et al. 2014), as well as contextual factors such as facial expression, expectations of reward or pain, and mood (Ellingsen et al. 2015).

More generally, parent-offspring attachment provides a rich repertoire of neural mechanisms and behaviors that can be extended to coordinate attachment in adult relationships, or between conspecifics that are more distantly related. This could be the case for endorphin/opioid-mediated "rubber band" mechanisms discussed above. It may also be the case for a candidate "glue-like" mechanism, involving the neuropeptide oxytocin, which is associated with a range of maternal behaviors (Uvnas-Moberg et al. 2014). Tactile stimulation is a major trigger for oxytocin release, especially for uterine contraction in labor, and milk ejection in lactation and breastfeeding. However, stimulation of sensory neurons outside a parturition context can also stimulate oxytocin release. Oxytocin can also have modulatory effects on neurotransmitter signaling in other neural systems, such as dopamine (Insel 2003) and serotonin (Yoshida et al. 2009) pathways. Oxytocin can play a stress-buffering role by dampening stress-related heart responses via noradrenergic neurons (Petersson and Uvnas-Moberg 2003), as well as influencing blood levels of the stress-related hormone cortisol (Rehn et al. 2014, Gamer and Buchel 2012).

Social touch interactions with family, partners, and friends are also associated with oxytocin release. Oxytocin circulating in the bloodstream during touch interactions between domestic dogs and their human caregivers increase significantly in both the dog and the human, on a scale of about 4-5 min (Rehn et al. 2014). When dogs and their caregivers are reunited after a separation, oxytocin levels rise in each, but the stress hormone cortisol in the dogs only begins to fall if the reunion includes touching, rather than merely visual and vocal contact (Rehn et al. 2014). Further, the OT rises in humans may have been anticipatory, with baseline rises preceding an impending touch interaction or reunion event.

Opioid and oxytocin systems are distinct, but in many respects they can function in a convergent or overlapping manner in social interactions. Convergent effects include subjective hedonic feelings, mild painkilling properties, an influence on HPA and SAM axes, as well as the capacity for fine-tuned context-sensitivity via cortical top-down modulation. Each is tightly associated with affiliative behaviors, particularly those involving touch - for example, the petting, nudging, grooming, play-elicitation, and snuggling that often accompanies a reunion in many species. The systems can also modulate each other, with beta-endorphins, secreted by the pituitary gland, interacting with oxytocin, secreted by the hypothalamus. Oxytocin is also tightly bound to reproductive cycles and seasonality - in human females endogenous release varies 
through the estrous cycle and has peaks surrounding birth and breastfeeding, and in meadow voles (Microtus pennsylvanicus), oxytocin receptor density varies seasonally (Beery et al. 2014). Stress-touch dynamics also show differential effects in males and females (Light et al. 2005), and nasally administered oxytocin may have sex-specific effects on affective touch (Scheele et al. 2014). But there is also evidence that these systems may interact in a complex manner, with gain-of-function MOR variants associated with higher parturition-related, but otherwise lower general levels, of circulating oxytocin in free-ranging macaques (Higham et al. 2011). So while the evidence indicates strong contributions of oxytocin and opioid systems to social touch behavior - especially in the context of pair bonding, birth, and nurturing - more investigation is needed into the effects of complex factors such species, sex, reproductive and offspring care strategies, and interaction with other key hormones and neurotransmitters.

\section{Prosocial Touch: Allogrooming and Consolation}

Despite being a potential source of stress (Sapolsky 2005), the social environment can also provide a rich fund of interactions that can offset the immediate costs of stress (Cohen and Wills 1985). Through the mechanisms outlined in the foregoing sections, social touch can buffer stress responses, and is also instrumental in proximityregulation and associated affiliative behavior. "Prosocial" touch takes these systems a step further. In humans and certain other species, individuals have the ability to intentionally confer the benefits of social, affective touch on others, without necessarily receiving an immediate benefit themselves. Prosocial touch can be defined as intentional social touch that benefits the "touchee" via physiological regulation of bodily responses. For example, holding someone's hand when they are experiencing the threat of pain can reduce anxiety (Coan et al. 2013). For most mammals, allogrooming (grooming the fur or skin of a conspecific) likely confers such benefits (Dunbar 2010; Aureli et al. 1999). Consoling touch - touch stimulation that is comforting, or touching actions that are intended to give comfort-represents another major form of prosocial touch in the mammalian repertoire (Clay and de Waal 2013).

Allogrooming has practical benefits with respect to parasite removal and hygiene (Dunbar 2010). However, in many primate species individuals spend more time allogrooming than mere hygiene would warrant. The role of allogrooming in primates has thus been proposed as a means of forming and maintaining relationships, rather than of keeping each other clean (Dunbar 2010). There is evidence from macaque monkeys that such allogrooming relationships influence the concentration in the central nervous system of beta-endorphins, one of the hormones that binds to MORs (Keverne et al. 1989). Because opioid modulation can have regulatory, hedonic, and behavioral effects, this could suggest that grooming can have "feel-good" consequences, though the effects of allogrooming and of relationships themselves are difficult to disentangle. Yet Homo sapiens may be an outlier species where allogrooming is concerned: we spend more time talking than allogrooming (Dunbar 2010), and culture imposes major constraints on touching (Suvilehto et al. 2015).

Oxytocin is also implicated in allogrooming, with higher levels in the blood and central nervous system of chimpanzees (Pan troglodytes) correlated with grooming (Maestripieri 
et al. 2009), and influenced by the strength of the relationship between wild chimpanzees (Crockford et al. 2013). Administration of a chemical that facilitates oxytocin receptor binding has been shown to increase allogrooming (Drago et al. 1986, Pedersen et al. 1988, Witt et al. 1992). Allogrooming by familiar humans in captive macaques has also been associated with decreases in heart rate (Grandi and Ishida 2015). After stressful approaches by dominant conspecifics, heart rate decreased faster when the macaque monkey received allogrooming than when it did not (Aureli et al. 1999). In domestically-reared lambs, gentle stroking by human caregivers slowed heart rate and was also associated with greater ear droopiness, an index of relaxation (Coulon et al. 2015).

Consolation may have comparable effects. Like humans, other primates have been observed to give tactile "contact comfort" to others after stressful events like fights (Palagi et al. 2006, Romero and de Waal 2010). This is referred to as consolation behavior, and is more likely to occur between socially close individuals in chimpanzees (Palagi et al. 2004, Romero and de Waal 2010), and related individuals in macaques (Aureli et al. 1997). Consolation may also reduce stress levels (Fraser et al. 2008). Humans and chimpanzees - but not capuchin monkeys - exhibit consolation behavior, and one explanation may be that consolation behavior requires an ability not only to take the other's emotional perspective, but to place value on taking prosocial action (Romero et al. 2010).

However, recent research suggests that consolation may not be the exclusive provenance of chimpanzees and humans, nor indeed of primates. Prairie voles (Microtus ochrogaster) increase grooming towards cagemates that have experienced a stressful event (Burkett et al. 2016), decreasing their stress behaviors. This may be mediated by oxytocin levels in the consoling vole's brain. Indeed, when both prairie voles (Burkett et al. 2016) and humans (Buchanan et al. 2012), observe a conspecific under stress, HPA stress response levels increase (as reflected by corticosterone in mice, and its equivalent cortisol in humans). In humans, offering to help a distressed patient decreased the observer's own distress, and even promoted a subjective "warm glow" (Preston et al. 2013). This evidence not only suggests that mere observation of stress can elevate HPA responses, but perhaps also provides a further motivational mechanism for touch-seeking or consolation behavior - if perceiving stress in others stresses us out, grooming or consoling the other may not only alleviate the other's stress, but may also be an avenue for physiological regulation of our own stress.

\section{Neural Bases of Stress Regulation by Social Touch}

The HPA, SAM, and thermoregulatory systems discussed in the foregoing sections share some of the same general neural circuitry in the brain, especially in phylogenetically more conserved (evolutionarily older) brainstem and basal forebrain nuclei. These circuits partly support other vital, often reflexive, allostatic processes such as maintaining stable relationships between sympathetic and parasympathetic nerve activity, as well as respiration, feeding, digestion, and other critical functions. Incoming touch and temperature pathways also have access to this circuitry. Crucially, the human neocortex exerts a comparatively large degree of influence on these subcortical systems (see eg Diehl 2005), allowing further levels of modulatory control. This section provides a sketch of how the neural architecture of social touch and attachment share fundamental pathways with the architecture of physiological regulation in response to challenge. These 
commonalities, as well as the opportunities for cortical modulation, imply that social attachment represents a form of flexible, context-dependent physiological regulation. Social relationships and interactions may therefore extend the reach of stress buffering systems to include critical mediators outside the individual's own physiology. This means that other humans are part of the equation in maintaining an energy-efficient allostatic balance in individual bodies.

In Fig. 2, major features of this circuitry have been distilled to present regions and pathways highly relevant to stress buffering and social touch. (It is coarse-grained in that different key systems can involve different populations within the same nuclei, while still participating in the same overall circuit organization.) Major nuclei in the hypothalamus and brainstem that serve as important hubs in allostatic regulation with specific regard to social touch are the paraventricular nucleus (PVN), and the arcuate nucleus (ARC), which are reciprocally connected with an important brainstem nucleus, the nucleus of the solitary tract (NTS).

The PVN and the ARC are hypothalamic nuclei which are tightly interconnected and contain oxytocin and opioid neurons. In particular, the PVN has dense oxytocin neurons, and is the major site of oxytocin release in the brain (Uvnas-Moberg et al. 2014). The ARC has dense opioid neurons, alongside neurons for other key neurotransmitters (Sapru 2013; Bali et al. 2015). Both the ARC and the PVN send and receive projections to and from the NTS, a brainstem nucleus that serves as a way station for allostatic regulatory influence on many basic bodily functions. In particular, the NTS contains neurons that modulate important HPA and SAM feedback loopsespecially those involving the neurotransmitter noradrenalin, and corticotropin releasing hormone (CRH), which stimulates cortisol release (Sapru 2013; Bali et al. 2015).

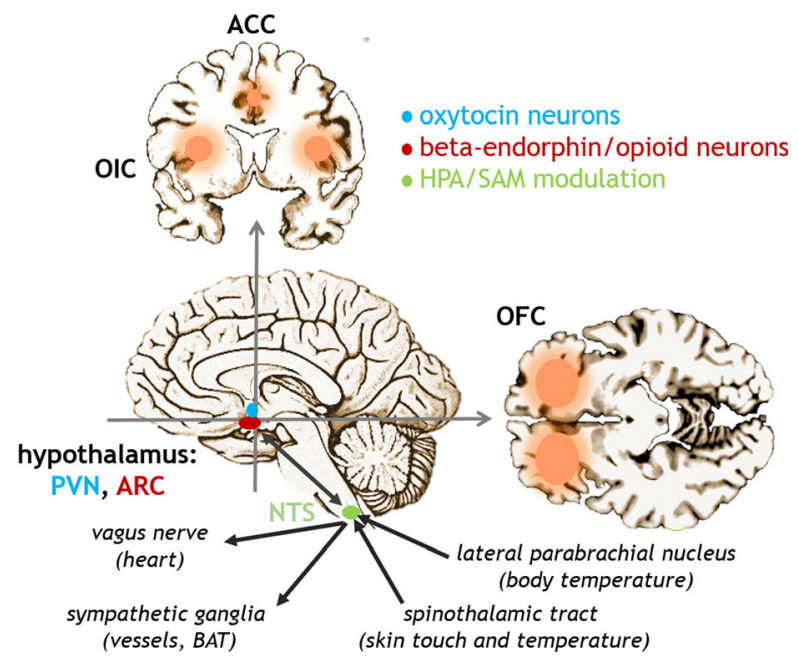

Fig. 2 A basic neurocircuitry of stress buffering by social touch. Major subcortical structures involved in a range of regulatory signaling influencing autonomic responses and other bodily systems share circuitry with touch-related responses. The paraventricular nucleus (PVN; blue) and the arcuate nucleus (ARC; red) are involved in beta-endorphin/mu-opioid mediated responses to social touch simulation, as well as stress responses. The nucleus of the solitary tract (NTS; green) receives projections from these nuclei as well as from touch-related signaling involving temperature changes and skin stimulation, and it influences heart and vascular responses via sympathetic modulation. Major cortical networks are also implicated in social touch, such as the OIC (opercoinsular cortex), ACC (anterior cingulate cortex), and OFC (orbitofrontal cortex) 
Notably, it is mainly NTS-PVN interactions involving oxytocin secretion which can inhibit or antagonize CRH via noradrenergic signaling (Meyer-Lindenberg et al. 2011, Uvnas-Moberg et al. 2014). The NTS also has major outputs to the vagal nerve, influencing heart rate, and to sympathetic ganglia, influencing blood pressure (Zoccal et al. 2014). It is also an important part of the pathway for endothermogenesis, influencing the metabolism of brown adipose fat (Morrison et al. 2008, Ryu et al. 2015). It receives signals about temperature and touch on the skin from $\mathrm{C}$ afferents via the spinothalamic tract (STT; (Campeau 2000) but see (Abraira and Ginty 2013), and core thermoreceptive information via the lateral parabrachial nucleus, LPB (Nakamura and Morrison 2008a, b). Oxytocin release in the NTS may even facilitate sensoryinduced oxytocin release in the PVN, creating a feedforward mechanism for the touchoxytocin relationship (Burbach et al. 1980, Burbach et al. 1992).

These subcortical hubs also have several important indirect connections with limbic and basal forebrain structures. The PVN is indirectly connected with the amygdala, which is sensitive to stress-related cues as well as socially-relevant facial expressions (Davis et al. 2016). The amygdala also secretes CRH during stress challenges, stimulating cortisol release; this in turn can trigger beta-endorphin release, exerting an inhibitory influence on the HPA axis (Nakagawasai et al. 1999, Bali et al. 2015). Binding of MOR receptors in the amygdala by beta-endorphins has been associated with more effective coping behavior in rats (Grisel et al. 2008, Barfield et al. 2010). Another subcortical site in this wider network is the nucleus accumbens, associated with motivation and reward (Floresco 2015), and which is indirectly connected with the ARC. The pituitary gland is another important subcortical hub, secreting betaendorphins and sending projections to the ARC (Bali et al. 2015).

Via such direct and indirect connections, cortical regions also exert a modulatory influence on these circuits. In prairie voles (Microtus ochrogaster) the anterior cingulate cortex (ACC) expresses oxytocin receptors (Burkett et al. 2016). The operculoinsular cortex encompasses posterior insula and somatosensory areas on nearby parietal operculum, which have been implicated in the processing of affective touch (Olausson et al. 2002, Morrison et al. 2011, McGlone et al. 2014, Morrison 2016a). Human neuroimaging evidence points to posterior insula as the prime cortical target of the CT pathway, alongside a high but less selective activation likelihood of secondary somatosensory cortex activation during affective touch (Morrison 2016a). For its part, the posterior insula is activated by a broad range of somatosensory stimulation in humans (Kurth et al. 2010, and is highly interconnected with parietal sensorimotor cortices (Cerliani et al. 2012). The orbitofrontal cortex has also been implicated in affective touch processing (McCabe et al. 2008, Francis et al. 1999), including primate allogrooming (Raleigh and Steklis 1981). The orbitofrontal cortex is associated with reward-related encoding and behavior (Padoa-Schioppa 2007; Li et al. 2016) and may work together with posterior insula to hedonically evaluate affective touch in ways that guide behavior. Behavioral thermoregulation has been associated with the posterior parietal cortex (Gallace et al. 2014).

The brainstem, subcortical, and cortical circuitry outlined here represents only a coarse sketch of the major brain areas and networks likely involved in any stress-buffering effects of social touch. It reflects a functional manifold underlying a wide range of autonomic and regulatory processes, tracing some of the main neural signaling pathways from the body to the brain, as well as regulatory "outflow" pathways from the brain back to the body, 
which operate in the service of allostasis. Considerably more is known about the roles of these systems in autonomic function and thermoregulation than in attachment behavior and social touch. However, in identifying key nodes such as the NTS, PVN, and the peripheral and cortical circuits they participate in, this sketch provides anchor points for further mapping of function-structure relationships relevant to social touch.

\section{Conclusion}

From this evidence, social touch emerges as being well-situated to act as a stress buffer, by virtue of multiple channels that converge on similar allostasis-related neurocircuitry and signaling pathways. Common to these neural pathways are brain networks that play various roles in regulating proximity to conspecifics. Each of these general systemssocial thermoregulation, social attachment via opioid and oxytocin signaling, and prosocial touch - may have their evolutionary origins in systems adapted for specific forms of parental nurturance and offspring attachment behavior. A more deeply underlying dynamic, especially in the manifestation of adaptive responses to stress challenges, is the efficient use of energy in the optimization of safety and in the juggling of life's challenges.

Acknowledgments Thanks to Stephanie Preston, Giovanni Novembre, and an anonymous reviewer for valuable comments on a previous draft.

Open Access This article is distributed under the terms of the Creative Commons Attribution 4.0 International License (http://creativecommons.org/licenses/by/4.0/), which permits unrestricted use, distribution, and reproduction in any medium, provided you give appropriate credit to the original author(s) and the source, provide a link to the Creative Commons license, and indicate if changes were made.

\section{References}

Abraira, V. E., \& Ginty, D. D. (2013). The sensory neurons of touch. Neuron, 79, 618-639.

Ackerley, R., Backlund Wasling, H., Liljencrantz, J., Olausson, H., Johnson, R. D., \& Wessberg, J. (2014). Human C-tactile afferents are tuned to the temperature of a skin-stroking caress. The Journal of Neuroscience, 34, 2879-2883.

Alberts, J. R. (2007). Huddling by rat pups: ontogeny of individual and group behavior. Developmental Psychobiology, 49, 22-32.

Aureli, F., Das, M., \& Veenema, H. C. (1997). Differential kinship effect on reconciliation in three species of macaques (Macaca fascicularis, M. fuscata, and M. sylvanus. Journal of Comparative Psychology, 111, 91-99.

Aureli, F., Preston, S. D., \& de Waal, F. B. (1999). Heart rate responses to social interactions in free-moving rhesus macaques (Macaca mulatta): a pilot study. Journal of Comparative Psychology, 113, 59-65.

Bali, A., Randhawa, P. K., \& Jaggi, A. S. (2015). Stress and opioids: role of opioids in modulating stressrelated behavior and effect of stress on morphine conditioned place preference. Neuroscience and Biobehavioral Reviews, 51, 138-150.

Barfield, E. T., Barry, S. M., Hodgin, H. B., Thompson, B. M., Allen, S. S., \& Grisel, J. E. (2010). Betaendorphin mediates behavioral despair and the effect of ethanol on the tail suspension test in mice. Alcoholism, Clinical and Experimental Research, 34, 1066-1072.

Barr, C. S., Schwandt, M. L., Lindell, S. G., Higley, J. D., Maestripieri, D., Goldman, D., Suomi, S. J., \& Heilig, M. (2008). Variation at the mu-opioid receptor gene (OPRM1) influences attachment behavior in infant primates. Proceedings of the National Academy of Sciences of the United States of America, 105, $5277-5281$. 
Beery, A. K., Vahaba, D. M., \& Grunberg, D. M. (2014). Corticotropin-releasing factor receptor densities vary with photoperiod and sociality. Hormones and Behavior, 66, 779-786.

Björnsdotter, M., Morrison, I., \& Olausson, H. (2010). Feeling good: on the role of C fiber mediated touch in interoception. Experimental Brain Research, 207, 149-155.

Blumberg, M. S., Efimova, I. V., \& Alberts, J. R. (1992). Ultrasonic vocalizations by rat pups: the primary importance of ambient temperature and the thermal significance of contact comfort. Developmental Psychobiology, 25, 229-250.

Buchanan, T. W., Bagley, S. L., Stansfield, R. B., \& Preston, S. D. (2012). The empathic, physiological resonance of stress. Social Neuroscience, 7, 191-201.

Burbach, J. P., Schotman, P., \& de Kloet, E. R. (1980). Oxytocin biotransformation in the rat limbic brain: chemical characterization of two oxytocin fragments and proposed pathway for oxytocin conversion. Biochemical and Biophysical Research Communications, 97, 1005-1013.

Burbach, J. P., Adan, R. A., \& de Bree, F. M. (1992). Regulation of oxytocin gene expression and forms of oxytocin in the brain. Annals of the New York Academy of Sciences, 652, 1-13.

Burbridge, S., Stewart, I., \& Placzek, M. (2016). Development of the neuroendocrine hypothalamus. Compr Physiol, 6, 623-643.

Burkett, J. P., Andari, E., Johnson, Z. V., Curry, D. C., de Waal, F. B., \& Young, L. J. (2016). Oxytocindependent consolation behavior in rodents. Science, 351, 375-378.

Campeau, S. and S. J. Watson, Jr. (2000). Connections of some auditory-responsive posterior thalamic nuclei putatively involved in activation of the hypothalamo-pituitary-adrenocortical axis in response to audiogenic stress in rats: an anterograde and retrograde tract tracing study combined with Fos expression. The Journal of Comparative Neurology 423: 474-491.

Castellani, J. W., Young, A. J., (2016). Human physiological responses to cold exposure: Acute responses and acclimatization to prolonged exposure. Autonomic Neuroscience, 196, 63-74.

Cerliani, L., Thomas, R. M., Jbabdi, S., Siero, J. C., Nanetti, L., Crippa, A., Gazzola, V., D'Arceuil, H., \& Keysers, C. (2012). Probabilistic tractography recovers a rostrocaudal trajectory of connectivity variability in the human insular cortex. Human Brain Mapping, 33, 2005-2034.

Champagne, D. L., Bagot, R. C., van Hasselt, F., Ramakers, G., Meaney, M. J., de Kloet, E. R., Joels, M., \& Krugers, H. (2008). Maternal care and hippocampal plasticity: evidence for experience-dependent structural plasticity, altered synaptic functioning, and differential responsiveness to glucocorticoids and stress. The Journal of Neuroscience, 28, 6037-6045.

Champagne, F. A., Curley, J. P., Swaney, W. T., Hasen, N. S., \& Keverne, E. B. (2009). Paternal influence on female behavior: the role of Peg3 in exploration, olfaction, and neuroendocrine regulation of maternal behavior of female mice. Behavioral Neuroscience, 123, 469-480.

Clay, Z., \& de Waal, F. B. (2013). Development of socio-emotional competence in bonobos. Proceedings of the National Academy of Sciences of the United States of America, 110, 18121-18126.

Coan, J. A., Kasle, S., Jackson, A., Schaefer, H. S., \& Davidson, R. J. (2013). Mutuality and the social regulation of neural threat responding. Attachment \& Human Development, 15, 303-315.

Cohen, S., Wills, T. A. (1985). Stress, social support, and the buffering hypothesis. Psychological Bulletin, 98(2), 310-357.

Coulon, M., Nowak, R., Peyrat, J., Chandeze, H., Boissy, A., \& Boivin, X. (2015). Do lambs perceive regular human stroking as pleasant? Behavior and heart rate variability analyses. PloS One, 10, e0118617.

Craig, A. D. (2003). Interoception: The sense of the physiological condition of the body. Current Opinion in Neurobiology, 13, 500-505.

Craig, A. D., \& Zhang, E. T. (2006). Retrograde analyses of spinothalamic projections in the macaque monkey: input to posterolateral thalamus. Journal of Comparative Neurology, 499, 953-964.

Crockford, C., Wittig, R. M., Langergraber, K., Ziegler, T. E., Zuberbuhler, K., \& Deschner, T. (2013). Urinary oxytocin and social bonding in related and unrelated wild chimpanzees. Proceedings of the Biological Sciences, 280, 20122765.

Davis, F. C., Neta, M., Kim, M. J., Moran, J. M., \& Whalen, P. J. (2016). Interpreting ambiguous social cues in unpredictable contexts. Social Cognitive and Affective Neuroscience, 11, 775-782.

Diehl, R. R. (2005). Vasovagal syncope and Darwinian fitness. Clinical Autonomic Research, 15, $126-129$.

Drago, F., Pedersen, C. A., Caldwell, J. D., \& Prange Jr., A. J. (1986). Oxytocin potently enhances noveltyinduced grooming behavior in the rat. Brain Research, 368, 287-295.

Dunbar, R. I. (2010). The social role of touch in humans and primates: behavioural function and neurobiological mechanisms. Neuroscience and Biobehavioral Reviews, 34, 260-268.

Ellingsen, D. M., Leknes, S., Loseth, G., Wessberg, J., \& Olausson, H. (2015). The neurobiology shaping affective touch: expectation, motivation, and meaning in the multisensory context. Frontiers in Psychology, 6, 1986. 
Farrell, W. J., \& Alberts, J. R. (2007). Rat behavioral thermoregulation integrates with nonshivering thermogenesis during postnatal development. Behavioral Neuroscience, 121, 1333-1341.

Floresco, S. B. (2015). The nucleus accumbens: an interface between cognition, emotion, and action. Annual Review of Psychology, 66, 25-52.

Francis, S., Rolls, E. T., Bowtell, R., McGlone, F., O'Doherty, J., Browning, A., Clare, S., \& Smith, E. (1999). The representation of pleasant touch in the brain and its relationship with taste and olfactory areas. Neuroreport, 10, 453-459.

Fraser, O. N., Stahl, D., \& Aureli, F. (2008). Stress reduction through consolation in chimpanzees. Proceedings of the National Academy of Sciences of the United States of America, 105, 8557-8562.

Gallace, A., Soravia, G., Cattaneo, Z., Moseley, G. L., \& Vallar, G. (2014). Temporary interference over the posterior parietal cortices disrupts thermoregulatory control in humans. PloS One, 9, e88209.

Gamer, M., \& Buchel, C. (2012). Oxytocin specifically enhances valence-dependent parasympathetic responses. Psychoneuroendocrinology, 37, 87-93.

Gilbert, C., Blanc, S., Giroud, S., Trabalon, M., Le Maho, Y., Perret, M., \& Ancel, A. (2007). Role of huddling on the energetic of growth in a newborn altricial mammal. American Journal of Physiology. Regulatory, Integrative and Comparative Physiology, 293, R867-R876.

Gilbert, C., McCafferty, D., Le Maho, Y., Martrette, J. M., Giroud, S., Blanc, S., \& Ancel, A. (2010). One for all and all for one: the energetic benefits of huddling in endotherms. Biological Reviews of the Cambridge Philosophical Society, 85, 545-569.

Gilbert, C., McCafferty, D. J., Giroud, S., Ancel, A., \& Blanc, S. (2012). Private heat for public warmth: how huddling shapes individual thermogenic responses of rabbit pups. PloS One, 7, e33553.

Gracheva, E. O., \& Bagriantsev, S. N. (2015). Evolutionary adaptation to thermosensation. Current Opinion in Neurobiology, 34, 67-73.

Grandi, L. C., \& Ishida, H. (2015). The physiological effect of human grooming on the heart rate and the heart rate variability of laboratory non-human primates: a pilot study in male rhesus monkeys. Front Vet Sci, 2, 50.

Grisel, J. E., Bartels, J. L., Allen, S. A., \& Turgeon, V. L. (2008). Influence of beta-endorphin on anxious behavior in mice: interaction with EtOH. Psychopharmacology, 200, 105-115.

Gunnar, M. R., \& Hostinar, C. E. (2015). The social buffering of the hypothalamic-pituitary-adrenocortical axis in humans: developmental and experiential determinants. Social Neuroscience, 10, 479-488.

Haim, A., Van Aarde, R. J., \& Skinner, J. D. (1992). Burrowing and huddling in newborn porcupine: the effect on thermoregulation. Physiology \& Behavior, 52, 247-250.

Herman, J. P., \& Cullinan, W. E. (1997). Neurocircuitry of stress: central control of the hypothalamo-pituitaryadrenocortical axis. Trends in Neurosciences, 20, 78-84.

Herman, J. P., Figueiredo, H., Mueller, N. K., Ulrich-Lai, Y., Ostrander, M. M., Choi, D. C., \& Cullinan, W. E. (2003). Central mechanisms of stress integration: hierarchical circuitry controlling hypothalamo-pituitary-adrenocortical responsiveness. Frontiers in Neuroendocrinology, 24, 151-180.

Higham, J. P., Barr, C. S., Hoffman, C. L., Mandalaywala, T. M., Parker, K. J., \& Maestripieri, D. (2011). Muopioid receptor (OPRM1) variation, oxytocin levels and maternal attachment in free-ranging rhesus macaques Macaca mulatta. Behavioral Neuroscience, 125, 131-136.

Hofer, M. A., Brunelli, S. A., \& Shair, H. N. (1993). The effects of 24-hr maternal separation and of litter-size reduction on the isolation-distress response of 12-day-old rat pups. Developmental Psychobiology, 26, 483-497.

Hostinar, C. E., Sullivan, R. M., \& Gunnar, M. R. (2014). Psychobiological mechanisms underlying the social buffering of the hypothalamic-pituitary-adrenocortical axis: a review of animal models and human studies across development. Psychological Bulletin, 140, 256-282.

IJzerman, H., Coan, J. A., Wagemans, F. M., Missler, M. A., van Beest, I., Lindenberg, S., \& Tops, M. (2015). A theory of social thermoregulation in human primates. Frontiers in Psychology, 21, 6-464.

Insel, T. R. (2003). Is social attachment an addictive disorder? Physiology \& Behavior, 79, 351-357.

Keverne, E. B., Martensz, N. D., \& Tuite, B. (1989). Beta-endorphin concentrations in cerebrospinal fluid of monkeys are influenced by grooming relationships. Psychoneuroendocrinology, 14, 155-161.

Kurth, F., Zilles, K., Fox, P. T., Laird, A. R., \& Eickhoff, S. B. (2010). A link between the systems: functional differentiation and integration within the human insula revealed by meta-analysis. Brain Structure \& Function, 214, 519-534.

Li, Y., Vanni-Mercier, G., Isnard, J., Mauguiere, F., \& Dreher, J. C. (2016). The neural dynamics of reward value and risk coding in the human orbitofrontal cortex. Brain, 139, 1295-1309.

Light, K. C., Grewen, K. M., \& Amico, J. A. (2005). More frequent partner hugs and higher oxytocin levels are linked to lower blood pressure and heart rate in premenopausal women. Biological Psychology, 69, 5-21.

Loken, L. S., Wessberg, J., Morrison, I., McGlone, F., \& Olausson, H. (2009). Coding of pleasant touch by unmyelinated afferents in humans. Nature Neuroscience, 12, 547-548. 
Loseth, G. E., Ellingsen, D. M., \& Leknes, S. (2014). State-dependent mu-opioid modulation of social motivation. Frontiers in Behavioral Neuroscience, 8, 430.

Lou, S., Duan, B., Vong, L., Lowell, B. B., \& Ma, Q. (2013). Runx1 controls terminal morphology and mechanosensitivity of VGLUT3-expressing C-mechanoreceptors. Journal of Neuroscience, 33, 870-882.

Ma, Q. (2010). Labeled lines meet and talk: population coding of somatic sensations. The Journal of Clinical Investigation, 120, 3773-3778.

Maestripieri, D., Hoffman, C. L., Anderson, G. M., Carter, C. S., \& Higley, J. D. (2009). Mother-infant interactions in free-ranging rhesus macaques: relationships between physiological and behavioral variables. Physiology \& Behavior, 96, 613-619.

McCabe, C., Rolls, E. T., Bilderbeck, A., \& McGlone, F. (2008). Cognitive influences on the affective representation of touch and the sight of touch in the human brain. Social Cognitive and Affective Neuroscience, 3, 97-108.

McEwen, B. S. (1998). Stress, adaptation, and disease. Allostasis and allostatic load. Annals of the New York Academy of Sciences, 840, 33-44.

McEwen, B. S. (2004). Protection and damage from acute and chronic stress: allostasis and allostatic overload and relevance to the pathophysiology of psychiatric disorders. Annals of the New York Academy of Sciences, 1032, 1-7.

McGlone, F., Wessberg, J., \& Olausson, H. (2014). Discriminative and affective touch: sensing and feeling. Neuron, 82, 737-755.

Meyer-Lindenberg, A., Domes, G., Kirsch, P., \& Heinrichs, M. (2011). Oxytocin and vasopressin in the human brain: social neuropeptides for translational medicine. Nature Reviews. Neuroscience, 12, 524-538.

Miller, A. H., Spencer, R. L., Hassett, J., Kim, C., Rhee, R., Ciurea, D., Dhabhar, F., McEwen, B., \& Stein, M. (1994). Effects of selective type I and II adrenal steroid agonists on immune cell distribution. Endocrinology, 135, 1934-1944.

Morrison, I. (2016a). ALE meta-analysis reveals dissociable networks for affective and discriminative aspects of touch. Human Brain Mapping, 37, 1308-1320.

Morrison, I. (2016b). CT afferent-mediated affective touch: brain networks and functional hypotheses. In $\mathrm{H}$. Olausson, I. Morrison, J. Wessberg, F. McGlone (Eds.), Affective touch and the neurophysiology of CT afferents. Amsterdam: Springer.

Morrison, S. F., Nakamura, K., \& Madden, C. J. (2008). Central control of thermogenesis in mammals. Experimental Physiology, 93, 773-797.

Morrison, I., Loken, L. S., \& Olausson, H. (2010). The skin as a social organ. Experimental Brain Research, 204, 305-314.

Morrison, I., Bjornsdotter, M., \& Olausson, H. (2011). Vicarious responses to social touch in posterior insular cortex are tuned to pleasant caressing speeds. The Journal of Neuroscience, 31, 9554-9562.

Nakagawasai, O., Tadano, T., Tan No, K., Niijima, F., Sakurada, S., Endo, Y., \& Kisara, K. (1999). Changes in beta-endorphin and stress-induced analgesia in mice after exposure to forced walking stress. Methods Find Exp Clin Pharmacol, 21, 471-476.

Nakamura, K., \& Morrison, S. F. (2008a). Preoptic mechanism for cold-defensive responses to skin cooling. The Journal of Physiology, 586, 2611-2620.

Nakamura, K., \& Morrison, S. F. (2008b). A thermosensory pathway that controls body temperature. Nature Neuroscience, 11, 62-71.

Nelson, E. E., \& Panksepp, J. (1998). Brain substrates of infant-mother attachment: contributions of opioids, oxytocin, and norepinephrine. Neuroscience and Biobehavioral Reviews, 22, 437-452.

Olausson, H., Lamarre, Y., Backlund, H., Morin, C., Wallin, B. G., Starck, G., Ekholm, S., Strigo, I., Worsley, K., Vallbo, A. B., \& Bushnell, M. C. (2002). Unmyelinated tactile afferents signal touch and project to insular cortex. Nature Neuroscience, 5, 900-904.

Olausson, H., Wessberg, J., Morrison, I., McGlone, F., \& Vallbo, A. (2010). The neurophysiology of unmyelinated tactile afferents. Neuroscience and Biobehavioral Reviews, 34, 185-191.

Osorio, C., Probert, T., Jones, E., Young, A. H., \& Robbins, I. (2016). Adapting to stress: understanding the neurobiology of resilience. Behavioral Medicine. doi:10.1080/08964289.2016.1170661.

Ouakinin, S. R. (2016). Anxiety as a risk factor for cardiovascular diseases. Front Psychiatry, 7, 25.

Padoa-Schioppa, C. (2007). Orbitofrontal cortex and the computation of economic value. Annals of the New York Academy of Sciences, 1121, 232-253.

Palagi, E., Paoli, T., \& Tarli, S. B. (2004). Reconciliation and consolation in captive bonobos (Pan paniscus. American Journal of Primatology, 62, 15-30.

Palagi, E., Cordoni, G., \& Borgognini Tarli, S. (2006). Possible roles of consolation in captive chimpanzees (Pan troglodytes. American Journal of Physical Anthropology, 129, 105-111. 
Panksepp, J. (1998). Affective neuroscience: the foundations of human and animal emotions. New York: Oxford University Press.

Panksepp, J., B. Herman, R. Conner, P. Bishop and J. P. Scott (1978a). The biology of social attachments: opiates alleviate separation distress. Biological Psychiatry 13: 607-618.

Panksepp, J., Vilberg, T., Bean, N. J., Coy, D. H., \& Kastin, A. J. (1978b). Reduction of distress vocalization in chicks by opiate-like peptides. Brain Research Bulletin, 3, 663-667.

Panksepp, J., Nelson, E., \& Bekkedal, M. (1997). Brain systems for the mediation of social separation-distress and social-reward. Evolutionary antecedents and neuropeptide intermediaries. Annals of the New York Academy of Sciences, 807, 78-100.

Pedersen, C. A., J. D. Caldwell, F. Drago, L. R. Noonan, G. Peterson, L. E. Hood and A. J. Prange, Jr. (1988). Grooming behavioral effects of oxytocin. Pharmacology, ontogeny, and comparisons with other nonapeptides. Annals of the New York Academy of Sciences 525: 245-256.

Petersson, M., \& Uvnas-Moberg, K. (2003). Systemic oxytocin treatment modulates glucocorticoid and mineralocorticoid receptor mRNA in the rat hippocampus. Neuroscience Letters, 343, 97-100.

Porges, S. W. (2007). The polyvagal perspective. Biological Psychology, 74, 116-143.

Prescott, S. A., Ma, Q., \& De Koninck, Y. (2014). Normal and abnormal coding of somatosensory stimuli causing pain. Nature Neuroscience, 17, 183-191.

Preston, S. D., Hofelich, A. J., Stansfield, R. B. (2013). The ethology of empathy: A taxonomy of realworld targets of need and their effect on observers. Frontiers in Human Neuroscience, 7, 488.

Raleigh, M. J., \& Steklis, H. D. (1981). Effect of orbitofrontal and temporal neocortical lesions of the affiliative behavior of vervet monkeys (Cercopithecus Aethiops Sabaeus. Experimental Neurology, 73, 378-389.

Rehn, T., Handlin, L., Uvnas-Moberg, K., \& Keeling, L. J. (2014). Dogs' endocrine and behavioural responses at Reunion are affected by how the human initiates contact. Physiology \& Behavior, 124, 45-53.

Romero, T., \& de Waal, F. B. (2010). Chimpanzee (Pan troglodytes) consolation: third-party identity as a window on possible function. Journal of Comparative Psychology, 124, 278-286.

Romero, T., Castellanos, M. A., \& de Waal, F. B. (2010). Consolation as possible expression of sympathetic concern among chimpanzees. Proceedings of the National Academy of Sciences of the United States of America, 107, 12110-12115.

Ryu, V., Garretson, J. T., Liu, Y., Vaughan, C. H., \& Bartness, T. J. (2015). Brown adipose tissue has sympathetic-sensory feedback circuits. The Journal of Neuroscience, 35, 2181-2190.

Sapolsky, R. M. (1994). The physiological relevance of glucocorticoid endangerment of the hippocampus. Annals of the New York Academy of Sciences, 746, 294-304.

Sapolsky, R. M. (2005). The influence of social hierarchy on primate health. Science, 308, 648-652.

Sapru, H. N. (2013). Role of the hypothalamic arcuate nucleus in cardiovascular regulation. Autonomic Neuroscience, $175,38-50$.

Scheele, D., Kendrick, K. M., Khouri, C., Kretzer, E., Schlapfer, T. E., Stoffel-Wagner, B., Gunturkun, O., Maier, W., \& Hurlemann, R. (2014). An oxytocin-induced facilitation of neural and emotional responses to social touch correlates inversely with autism traits. Neuropsychopharmacology, 39, 2078-2085.

Schirmer, A., Jesuthasan, S., \& Mathuru, A. S. (2013). Tactile stimulation reduces fear in fish. Frontiers in Behavioral Neuroscience, 7, 167.

Schulkin, J. (2011). Social allostasis: anticipatory regulation of the internal milieu. Frontiers in Evolutionary Neuroscience, 2, 111.

Seth, A. K., \& Critchley, H. D. (2013). Extending predictive processing to the body: emotion as interoceptive inference. The Behavioral and Brain Sciences, 36, 227-228.

Sterling, P. A. E. J. (1988). Allostasis: A new paradigm to explain arousal pathology. Handbook of Life Stress, Cognition and Health, John Wiley \& Sons, New York.

Suvilehto, J. T., Glerean, E., Dunbar, R. I., Hari, R., \& Nummenmaa, L. (2015). Topography of social touching depends on emotional bonds between humans. Proceedings of the National Academy of Sciences of the United States of America, 112, 13811-13816.

Troisi, A., Frazzetto, G., Carola, V., Di Lorenzo, G., Coviello, M., D'Amato, F. R., Moles, A., Siracusano, A., \& Gross, C. (2011). Social hedonic capacity is associated with the A118G polymorphism of the mu-opioid receptor gene (OPRM1) in adult healthy volunteers and psychiatric patients. Social Neuroscience, 6, 88-97.

Uvnas-Moberg, K., Handlin, L., \& Petersson, M. (2014). Self-soothing behaviors with particular reference to oxytocin release induced by non-noxious sensory stimulation. Frontiers in Psychology, 5, 1529.

Valentino, R. J., \& Van Bockstaele, E. (2015). Endogenous opioids: the downside of opposing stress. Neurobiol Stress, 1, 23-32.

Vallbo, A. B., Olausson, H., \& Wessberg, J. (1999). Unmyelinated afferents constitute a second system coding tactile stimuli of the human hairy skin. Journal of Neurophysiology, 81, 2753-2763. 
Wessberg, J., Olausson, H., Fernstrom, K. W., \& Vallbo, A. B. (2003). Receptive field properties of unmyelinated tactile afferents in the human skin. Journal of Neurophysiology, 89, 1567-1575.

Witt, D. M., Winslow, J. T., \& Insel, T. R. (1992). Enhanced social interactions in rats following chronic, centrally infused oxytocin. Pharmacology, Biochemistry, and Behavior, 43, 855-861.

Yoshida, M., Takayanagi, Y., Inoue, K., Kimura, T., Young, L. J., Onaka, T., \& Nishimori, K. (2009). Evidence that oxytocin exerts anxiolytic effects via oxytocin receptor expressed in serotonergic neurons in mice. The Journal of Neuroscience, 29, 2259-2271.

Zoccal, D. B., Furuya, W. I., Bassi, M., Colombari, D. S., \& Colombari, E. (2014). The nucleus of the solitary tract and the coordination of respiratory and sympathetic activities. Frontiers in Physiology, 5, 238. 